\title{
Use of a Novel Variable Power Radiofrequency Ablation System Specific for Knee Chondroplasty: Surgical Experience and Two-Year Patient Results
}

\author{
Danielle Piper ${ }^{1}$, Clare Taylor $^{1}$, Nick Howells ${ }^{1}$, James Murray ${ }^{1,2}$, Andrew Porteous ${ }^{1}$, James R. Robinson ${ }^{1}$ \\ 1. Trauma and Orthopaedics, Avon Orthopaedic Centre, Bristol, GBR 2. Trauma and Orthopaedics, North Bristol NHS \\ Trust, Bristol, GBR
}

Corresponding author: James R. Robinson, jrrobinson@doctors.org.uk

\section{Abstract \\ Introduction}

Although stabilisation of knee cartilage lesions (chondroplasty) may be performed with an arthroscopic shaver, more recently, radiofrequency (RF) ablation has gained in popularity. However, their remain some concerns about the avoidance of thermal injury, chondrolysis, and osteonecrosis with the use of RF devices.

\section{Methods}

We reviewed the outcomes of 85 knee chondroplasties performed with a new RF ablation wand designed for knee chondroplasty. Lesion details and Chondropaenia Severity Score (CSS) were recorded for each patient. We evaluated the occurrence of adverse outcomes, post-operative complications, and the need for further surgery. Post-operative outcomes scores (Oxford Knee Score [OKS], Knee injury and Osteoarthritis Outcome Score [KOOS], and International Knee Documentation Committee [IKDC] subjective knee outcome) were recorded at a minimum of one-year follow-up.

\section{Results}

At the final mean follow-up of 27.5 months (range: 12-46.6 months), 12 (14\%) knees had undergone or were listed for further surgery. Four patients had corticosteroid injections for ongoing pain at a median 7.5 months (range: 5-20 months) post-operatively. There were no observed re-operations considered to be caused by complications related to thermal injury. Of the six patients listed for or undergoing knee arthroplasty, five (83\%) had grade 4 lesions found at the arthroscopic chondroplasty. A negative correlation was noted between CCS, and post-operative IKDC subjective score $(R=-0.35)$, KOOS Sports $(R=-0.39)$, and KOOS QoL $(\mathrm{R}=-0.36)$.

\section{Conclusions}

We found that RF chondroplasty appeared safe, and there were no concerns with regard to thermal injury.

Review began 01/10/2021 Review ended 01/18/2021 Published 01/22/2021

\section{() Copyright 2021}

Piper et al. This is an open access article distributed under the terms of the Creative Commons Attribution License CC-BY 4.0., which permits unrestricted use, distribution, and reproduction in any medium, provided the original author and source are credited.
Functional outcome appeared to be related to the quality of chondral and meniscal tissue throughout all knee compartments, with better results for isolated grade 2 and 3 cartilage lesions.

\section{Categories: Orthopedics}

Keywords: chondroplasty, knee arthroscopy, radiofrequency ablation

\section{Introduction}

Focal articular surface lesions in the knee are common [1] and observed in approximately two-thirds of knee arthroscopies [2]. Chondral lesions may result from ageing, disease, and injury [3], often following a deleterious course [4] with progression in size and grade due to mechanical wear [5]. As a known precursor of osteoarthritis, untreated lesions may result in loss of function, pain, and swelling [6-8]. This may eventually lead to the requirement for knee arthroplasty or osteotomy. Given the risk of debilitating outcomes, intervention to stabilise chondral lesions may be warranted in an attempt to slow progression, maintain quality of life, and potentially delay more invasive surgery.

The most common of these interventions is arthroscopic chondroplasty [9], in which loose and fibrillated articular cartilage is removed in an attempt to smooth and stabilise the defect [10,11]. An arthroscopic shaver is often used to mechanically debride the lesion. However, this has been associated with inadvertent removal of adjacent healthy tissue and resultant lesion progression [12,13]. Radiofrequency (RF) ablation is an alternative that has recently gained in popularity for chondroplasty in arthroscopic knee surgery. Activation of the RF electrosurgical wand in a conductive solution (sodium chloride) causes a nonequilibrium plasma vapour layer to form at the tip of the bipolar electrode [14,15]. Current across the vapour layer produces electrons that break down the water molecules into highly energised free radicals, causing 
disintegration of organic bonds in adjacent tissue and forming gaseous ablation by-products [14]. Studies have suggested that bipolar RF ablation may be advantageous in achieving a smoother surface [16,17], with favourable results in trials comparing its use to that of a shaver [18-20]. However, there have been concerns about thermal injury [21], including chondrocyte death [22] and the development of post-operative chondrolysis and osteonecrosis [23].

Critical in achieving smooth, volumetric tissue removal, with a minimal margin of chondrocyte injury, is the ability to ablate target cartilage tissue with a stable plasma vapor layer around the bipolar electrode [14,24]. Maintenance of a stable plasma involves a complex interaction between current and flow of saline past the tip of the wand. This can be influenced by the wand being connected to the flow pump and control unit in the operating room. Additionally, with older generations of wands, it was necessary to activate the wand away from tissue to avoid thermal injury and then introduce the plasma vapour to target tissue by "hovering" above the cartilage surface in order to ablate it whilst avoiding pressure of the wand against the cartilage surface that could result in thermal injury [22].

In 2014, the Werewolf Generator and Flow 50 plasma wand (Smith and Nephew, Austin, TX, USA) were introduced to our department as part of a limited commercial release to replace an existing RF ablation system already in use. This device was developed with specific utility for knee chondroplasty with ablation modes adapted for specific tissue types (such as cartilage, meniscus, or synovium) and introduced new software to modulate the pump flow for increased plasma stability and ablation control. There are currently limited studies on the safety and functional performance of chondroplasty in large patient series. Novel technologies require studying in the clinical setting to ensure safety and gauge their real-world application. We hypothesised that the use of RF ablation for chondroplasty, when performed alone or in combination with meniscectomy or synovectomy, would be safe without complications such as thermal injury. We also wished to ascertain whether the overall quality of chondral and meniscal tissue throughout all knee compartments was related to post-operative outcome.

\section{Materials And Methods}

We identified patients from our research database who had undergone arthroscopic chondroplasty using the Werewolf generator and Flow 50 Coblation wand during a three-year period beginning from July 2014, when the device was introduced. The study was registered with our Local Patient Safety Assurance and Audit Clinical Governance Department (project number CE52507). Inclusion criteria were all patients undergoing knee arthroscopic chondroplasty with or without treatment of a meniscal lesion or synovectomy. Exclusions included associated ligament reconstruction and high tibial osteotomy. Patients with a chondral lesion stabilised using RF chondroplasty and then further treated with an additional cartilage procedure, such as microfracture or autologous matrix-induced chondrogenesis, were included. We used the electronic patient record (Bluespier, Droitwich, Worcestershire, UK) to review the operative notes and clinic letters to retrieve clinical information, the types of pathology treated, and whether any intra-operative complications were reported. Patient demographic data (age, sex, side of surgery), operative data (location, size, and grade of chondral of lesions), meniscal pathology, and treatment were recorded. In addition, during the study period, the operating surgeons were asked to record any intra-operative adverse event.

\section{Surgical technique}

The arthroscopic procedures were undertaken by seven fellowship-trained consultant knee surgeons working in our department. After initial inspection of the joint, meniscal and chondral lesions were addressed. The chondral surfaces were probed and graded using the Outerbridge score [25]. The Chondropaenia Severity Score (CSS) [26], representing the quality of chondral and meniscal tissue throughout all knee compartments, was calculated for each patient. Chondral lesions with unstable flaps at their periphery were treated by removal of the flaps to create a smooth stable margin at the periphery of the lesion. Areas of chondral fibrillation were smoothed.

The Werewolf generator and Flow 50 Coblation wand is designed specifically for use in the knee with variable power and flow settings. The generator has five operating modes that adjust the power and flow at the tip of the wand for use with different tissue types. The "Low" setting is indicated for use on articular cartilage. The wand has a 45-degree ceramic bevel at its tip (Figure 1) that may rest against the articular surface without the electrode contacting it, thus aiding the precision to the depth of tissue removal. The wand was activated and moved continuously with the aim of smoothing the flaps at the periphery of the chondral lesion. Both the intra-articular fluid and outflow temperatures were monitored within the hand piece. The controller was set to emit an alarm if the temperature exceeded $30^{\circ} \mathrm{C}$. Once the chondroplasty was performed, the chondral surface was probed to ensure smooth and stable margins at the periphery of the lesion. 


\section{Cureus}

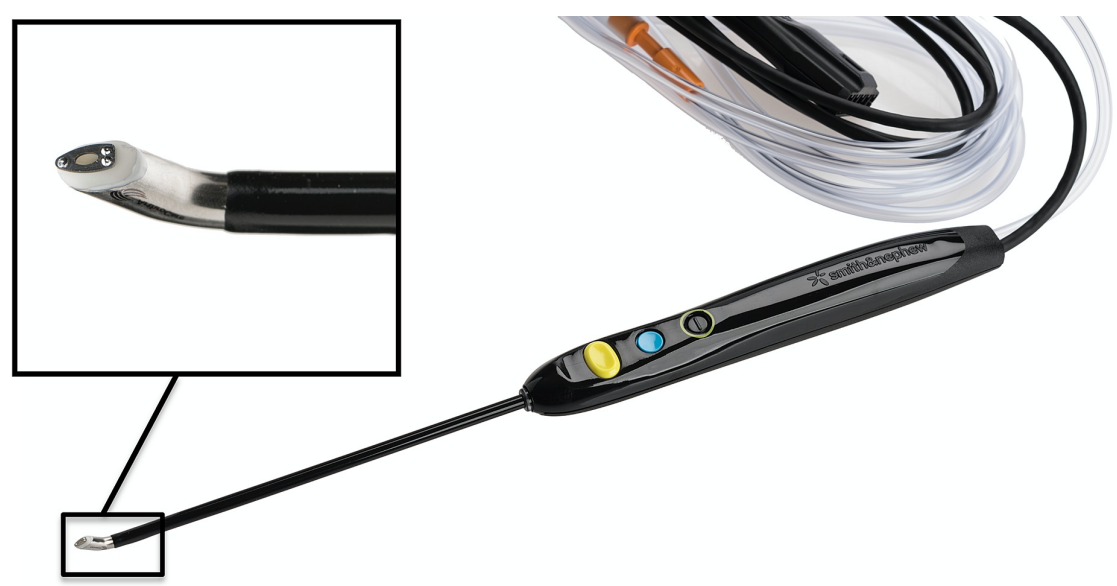

FIGURE 1: The Flow 50 RF ablation wand. The ceramic bevel at the tip of the wand allows the tip to rest on the joint surface without the electrode contacting the tissue. This design feature is aimed at improving the controlled introduction of target cartilage tissue into the stable plasma vapour layer adjacent to the bipolar electrode.

$\mathrm{RF}$, radiofrequency

\section{Study outcomes}

The primary outcome measures were the requirement for further surgery and complications related to the use of RF chondroplasty or surgery. The following patient-reported outcome measures (PROMs) were collected for all patients with a minimum 12-month follow-up as secondary outcome measures: Oxford Knee Score (OKS), Knee injury and Osteoarthritis Outcome Score (KOOS), and International Knee Documentation Committee (IKDC) subjective knee outcome. Patients were questioned about further operative interventions following RF chondroplasty and further details obtained from the electronic patient record. All patients were contacted by post with a letter explaining the study and providing PROMs questionnaires with a return, stamped envelope enclosed. Patients who had not returned completed outcome scores within two weeks were contacted by telephone. Three attempts were made to contact the patients by telephone before being documented as lost to follow-up.

\section{Statistical analysis}

All scores were entered into an Excel spreadsheet (Microsoft, Redmond, WA, USA), which was used to perform the statistical analysis. Student's t-test was used to compare differences in post-operative IKDC score between CCS grades. Pearson's correlation coefficient was used to assess factors associated with patient-reported outcome scores. A p-value of $<0.05$ was considered statistically significant.

\section{Results}

A total of 82 (28 females, 54 males; mean age: 41 years; range: 16-67 years) patients identified from the database met the inclusion criteria. Three patients underwent bilateral procedures. The details of the 85 operative procedures undertaken are provided in Table 1. 


\section{Cureus}

\section{Procedure}

Isolated chondroplasty

Chondroplasty and meniscectomy

Chondroplasty and meniscus repair

Chondroplasty and removal of loose body

Chondroplasty and microfracture/ autologous matrix-induced chondrogenesis

Chondroplasty and ACL graft plasty

Chondroplasty, meniscectomy, and removal of loose body

\section{TABLE 1: Operative procedures $(n=85)$}

$\mathrm{ACL}$, anterior cruciate ligament

\section{Chondral lesions treated}

Of the study cohort, 55 (67.1\%) patients had one chondral lesion treated, 25 (30.5\%) had two chondral lesions treated, and two (2.5\%) had three lesions. The mean CSS of the cohort was 83.5 (standard deviation = 9.8; range $=58-97$ ). The breakdown of chondral lesions treated is shown in Table 2.

\begin{tabular}{|l|l|l|}
\hline & Number of chondral lesions treated & Mean lesion size \\
\hline CSS grade A (85-100 points), $\mathrm{n}=34$ & 1 lesion (82\%); 2 lesions (18\%) & $190 \mathrm{~mm}^{2}$ \\
\hline CSS grade B (71-84 points), $\mathrm{n}=40$ & 1 lesion (67.5\%); 2 lesions (30\%); 3 lesions (2.5\%) & $304 \mathrm{~mm}^{2}$ \\
CSS grade C (56-70 points), $\mathrm{n}=8$ & 2 lesions (88\%); 3 lesions (12\%) & $237 \mathrm{~mm}^{2}$ \\
\hline
\end{tabular}

\section{TABLE 2: Cartilage lesions treated by CSS score}

CSS, Chondropaenia Severity Score

\section{Further interventions and complications}

At the final mean follow-up of 27.5 months (range: 12-46.6 months), 12 knees had undergone or were listed for further surgery: five total knee replacement, one patellofemoral joint replacement, three high tibial osteotomies, and three arthroscopies (Table 3). Four patients had corticosteroid injections for ongoing pain at a median of 7.5 months post-operatively (range: 5-20 months). 


\section{Cureus}

Patient demographics

Details of primary surgery

A 55-year-old female

A 55-year-old female

A 67-year-old male

A 50-year-old

male

A 47-year-old male

A 45-year-old female

A 32-year-old

male

A 23-year-old female

A 30-year-old male

A 23-year-old male

A 32-year-old male

Chondroplasty of $240 \mathrm{~mm}^{2}$ grade IV medial femoral condyle lesion and medial tibial plateau lesion. Degenerative medial meniscus tear resected

Chondroplasty of $200 \mathrm{~mm}^{2}$ grade II medial femoral condyle lesion and $100 \mathrm{~mm}^{2}$ grade III lateral tibial plateau lesion

Chondroplasty of $400 \mathrm{~mm}^{2}$ grade IV trochlear lesion and grade II lateral femoral condyle lesion

Chondroplasty of $150 \mathrm{~mm}^{2}$ grade IV medial femoral condyle lesion and $180 \mathrm{~mm}^{2}$ grade IV trochlear lesion

Chondroplasty of $200 \mathrm{~mm}^{2}$ grade IV medial femoral condyle lesion and $400 \mathrm{~mm}^{2}$ grade IV trochlear lesion

Chondroplasty of $400 \mathrm{~mm}^{2}$ grade IV trochlear lesion and $400 \mathrm{~mm}^{2}$ grade IV patella lesion

Chondroplasty of chondral flaps around previous osteochondral mosaicplasty of the medial femoral condyle

Chondroplasty of $100 \mathrm{~mm}^{2}$ grade IV chondral lesion and lateral femoral condyle lesion

Chondroplasty of $420 \mathrm{~mm}^{2}$ grade IV medial femoral condyle chondral lesion

Chondroplasty of $25 \mathrm{~mm}^{2}$ medial femoral condyle chondral lesion and medial meniscus repair

Chondroplasty of $400 \mathrm{~mm}^{2}$ osteochondritis dissecans lesion in the medial femoral condyle

A 54-year-old

male
Further surgery (post-

operative month)

TKR (24 months)

TKR (at another institution, month not available)

TKR (23 months)

Listed for TKR (21 months)

TKR (7 months)

Listed for PFJ replacement (38 months)

Listed for HTO (46 months)

Medial closing wedge HTO (9 months)

Medial opening wedge HTO (12 months)

Arthroscopy for further medial meniscal tear (10 months)

Listed for further arthroscopic chondral surgery

Arthroscopic washout of haematoma (2 weeks)

TABLE 3: Details of further surgery $(n=12)$

HTO, high tibial osteotomy; PFJ, patellofemoral joint replacement; TKR, total knee replacement

There were no observed re-operations considered to be caused by complications related to the study device. One intra-operative complication was noted: the tip of the electrode dissociated from the wand. This was immediately identified, the tip was retrieved, and the wand and broken electrode were returned to the manufacturer for investigation. No obvious defects were found; it was presumed that the tip of the electrode might have been damaged when introduced the knee. No other safety concerns were reported by the operating surgeons. Additionally, in the subsequent four total knee arthroplasties performed at our institution, there were no findings suggestive of chondrolysis or osteonecrosis.

\section{PROMs}

At the final follow-up, the following PROMs were obtained: 42 replies for the OKS and 44 replies for the KOOS and IKDC (Table 4). Two patients failed to complete the OKS fully and did not respond to further attempts to provide complete scores. Also, 37 (32\%) patients did not answer the three follow-up calls or hung up, seven (8\%) did not have their scores taken because they had undergone additional surgery, and four (5\%) declined to complete PROMs scores but reported that their knee was functioning well. 


\section{Cureus}

\begin{tabular}{|l|l|l|l|l|l|}
\hline & N & Mean & Range & Median & Standard deviation \\
\hline OKS $(0=$ worst; $48=$ best $)$ & 42 & 35 & $7-48$ & 38 & 11 \\
KOOS Symptoms $(0=$ worst; $100=$ best $)$ & 44 & 64 & $0-100$ & 64 & 26 \\
KOOS Pain ( $0=$ worst; $100=$ best $)$ & 44 & 68 & $3-100$ & 69 & 24 \\
KOOS ADL ( $0=$ worst; $100=$ best) & 44 & 77 & $4-100$ & 82 & 23 \\
KOOS Sports $(0=$ worst; $100=$ best) & 44 & 52 & $0-100$ & 53 & 31 \\
KOOS QoL $(0=$ worst; $100=$ best) & 44 & 45 & $0-100$ & 44 & 29 \\
KOOS Total $(0=$ worst; $100=$ best $)$ & 44 & 61 & $5-100$ & 61 & 24 \\
IKDC (0=worst; $100=$ best) & 44 & 55 & $8-98$ & 56 & 24 \\
\hline
\end{tabular}

\section{TABLE 4: Post-operative PROMs data}

ADL, activities of daily living; IKDC, International Knee Documentation Committee; KOOS, Knee injury and Osteoarthritis Outcome Score; OKS, Oxford Knee Score; PROM, patient-reported outcome measure

A statistically significant negative correlation was noted between CCS and post-operative IKDC subjective score $(R=-0.35)$, KOOS Sports $(R=-0.39)$, and KOOS QoL ( $R=-0.36)$ (Table 5). No statistically significant correlation was found between CCS score and the other KOOS domains or the OKS. No statistically significant correlation was found between lesion size, number, age, and outcome score. There were no significant differences in post-operative PROM scores between patients undergoing isolated chondroplasty and those undergoing combined chondroplasty and meniscectomy.

\begin{tabular}{|c|c|c|c|}
\hline \multirow[b]{2}{*}{ PROMs } & \multicolumn{3}{|l|}{ CSS grade } \\
\hline & A & B & $\mathrm{C}$ \\
\hline OKS ( $0=$ worst; $48=$ best) & 42 (range 20-48; SD = 9) & 35 (range 7-47; SD = 10) & 28 (range $7-44 ; \mathrm{SD}=11$ ) \\
\hline KOOS Symptoms ( $0=$ worst; $100=$ best $)$ & 80 (range $30-100 ;$ SD = 24) & 65 (range 0-100; SD = 25) & 50 (range $0-75 ; \mathrm{SD}=21$ ) \\
\hline KOOS Pain (0=worst; $100=$ best) & 86 (range $28-100 ;$ SD = 20) & 68 (range 3-100; SD = 24) & 61 (range 20-48; SD = 9) \\
\hline KOOS ADL ( $0=$ worst; $100=$ best) & 94 (range 29-100; SD = 17) & 78 (range 4-100; SD = 25) & 71 (range 20-48; SD = 9) \\
\hline KOOS Sports ( $0=$ worst; $100=$ best $)$ & 75 (range 35-100; SD = 27) & 45 (range $0-85 ; \mathrm{SD}=30$ & 35 (range 0-48; SD = 9) \\
\hline KOOS QoL ( $0=$ worst, $100=$ best) & 56 (range 31-100; SD = 26) & 28 (range $0-81 ; \mathrm{SD}=27$ ) & 19 (range $0-62 ; \mathrm{SD}=25$ ) \\
\hline IKDC ( $0=$ worst: $100=$ best $)$ & 78 (range 51-99; SD = 16) & 55 (range $8-84: S D=19$ ) & $42($ range $8-70 ; S D=18)$ \\
\hline
\end{tabular}

\section{TABLE 5: Median PROMs scores by Chondropaenia Severity Score}

ADL, activities of daily living; CSS, Chondropaenia Severity Score; IKDC, International Knee Documentation Committee; KOOS, Knee injury and Osteoarthritis Outcome Score; QoL, quality of life; OKS, Oxford Knee Score; PROM, patient-reported outcome measure

\section{Discussion}

There are limited studies in the literature on RF ablation chondroplasty in the knee. The U.K. National Institute of Health and Care Excellence (NICE) 2014 guidance [21] recommended that there appeared to be short-term benefits, although clinicians should pay particular attention to the avoidance of thermal injury. It was stated that there was a need for further research and in particular that there was insufficient evidence for the use of arthroscopic RF chondroplasty in older patients with osteoarthritis. Our study reinforces these recommendations and found that chondroplasty using the Flow 50 plasma ablation wand and Werewolf generator, particularly for patients with higher CSS (indicating better quality of chondral and meniscal tissue throughout all knee compartments), appeared safe for the treatment of small, isolated chondral defects.

RF ablation as an alternative to mechanical debridement has recently gained in popularity for chondroplasty in arthroscopic knee surgery. Studies have suggested that, compared with the use of a mechanical shaver, a 
macroscopically smoother surface may be obtained [16,17,22,27] with reduced cartilage permeability and maintenance of cartilage structural properties [28]. In a randomised controlled clinical study comparing the use of RF ablation to a mechanical shaver for chondroplasty at the time of meniscectomy [18-20], PROMs were improved and there were fewer re-operations. Voloshin et al. [29] undertook second-look arthroscopies two years following RF ablation chondroplasty and found that the majority (88\%) of lesions showed no sign of progression and 56\% appeared improved with complete or partial filling. Although there have been concerns about thermal injury to cartilage with bipolar RF [17,22,23], Amiel et al. [24] found that with optimal wand usage, thermal penetration in the treatment area may be minimised, limiting chondrocyte death to approximately $125 \mu \mathrm{m}$ compared with approximately $250 \mu \mathrm{m}$ with a mechanical shaver.

Maintaining a stable plasma vapour layer was challenging with older RF wand generations. Operating room suction units can cause too much suction at the tip of the bipolar electrode to allow stable plasma formation, and the tip of the wands has to be held "hovering" above the cartilage surface in order to introduce the plasma vapour layer yet avoid pressure of the wand against the cartilage and subsequent thermal injury. Despite this, chondroplasty series with older wand generations have shown acceptable results $[10,11,15,19]$.

It is important to monitor all new surgical techniques and devices as they are introduced into clinical practice, particularly due to the concerns regarding the risk of thermal damage to surrounding cartilage when using RF within the knee. Our study demonstrated device safety with PROMs results at a mean followup of 27.5 months, which is comparable to other case series of RF chondroplasty with much shorter followup (three months) [15]. None of the surgeons reported safety concerns, and we found no evidence of concerns with thermal injury that have been reported in other series [23].

There were a number of re-operations in our cohort of patients. Of the patients who went onto joint arthroplasty, five out of the six had large grade 4 chondral lesions found at surgery. In these cases, arthroscopic assessment of the knee was undertaken where pre-operative imaging had failed to demonstrate full-thickness chondral lesions or to determine suitability for uni-compartmental arthroplasty. In some cases, there had been progression of the cartilage lesion whilst the patient had been on a surgical waiting list. In these cases, which were found to have lower CSS, the outcomes were poor. RF chondroplasty is not indicated for grade 4 disease when this is part of established osteoarthritis and the minimal efficacy of arthroscopic procedures, where there is advanced degenerative change, is increasingly understood [30]. Although previous comparative studies of the outcomes of RF chondroplasty have excluded patients with grade 4 lesions [10,11], our series suggests that for small, isolated grade 4 chondral lesions, RF chondroplasty may be reasonable in order to stabilise them and to ascertain suitability for further biological treatment or focal resurfacing.

This study is limited by its retrospective design and the lack of pre-operative PROM scores. Additionally, we did not undertake a comparison between different types of RF wand. However, our study does allow comparison of post-operative outcomes with previously published chondroplasty cohorts. Gharaibeh et al. [15] retrospectively analysed a cohort of patients undergoing chondroplasty with an older generation of RF ablation device and reported a statistically significant improvement in normalised total KOOS from a mean pre-operative score of 45.9 (SD: 16.8) to mean post-operative score of 65.6 (SD: 18.9) at an average follow-up of 4.2 months post-operatively. The mean post-operative total KOOS score of 61.3 (SD: 24 ) we found in our study, at much longer-term follow-up (mean: 27.5 months), compares reasonably well, particularly as KOOS scores following chondroplasty are known to diminish from one-year post-operative [18]. Unlike Gharaibeh et al. we did not find poorer outcomes in those patients undergoing combined meniscectomy and chondroplasty, although we found a lower CSS score was associated with poorer post-operative PROMs.

\section{Conclusions}

The Werewolf/Flow 50 system for RF knee chondroplasty appears safe within routine clinical practice, with no specific concerns with regard to thermal injury. Functional post-operative outcomes relate to the quality of chondral and meniscal tissue throughout all knee compartments. Correlation between higher CSS at surgery and better post-operative function (IKDC subjective score and KOOS sports and quality-of-life domains) further supports the recommendation that RF chondroplasty is better suited for the stabilisation of discreet chondral lesions in knees with minimal deterioration in other compartments. There is decreased utility in patients with osteoarthritis, particularly if widespread. Further work is needed to assess the longterm outcomes of RF chondroplasty with this system.

\section{Additional Information}

\section{Disclosures}

Human subjects: Consent was obtained by all participants in this study. Animal subjects: All authors have confirmed that this study did not involve animal subjects or tissue. Conflicts of interest: In compliance with the ICMJE uniform disclosure form, all authors declare the following: Payment/services info: Research Grant funding from Smith and Nephew . Financial relationships: James Robinson - Consulting, Lectures Smith and Nephew, Grant - Newclip Technics, Conmed - Consulting, Lectures, Arthrex - Consulting, 
Lectures Dani Piper - no conflict Clare Taylor - no conflict James Murray - Smith and Nephew - Consulting, Lectures Andrew Porteous - Msith and Nephew - Consulting, Lectures, Zimmer Biomet - Royalties Institutional funding from Smith and Nephew, Newclip Technics declare(s) a grant, personal fees, a patent and royalties from Smith and Nephew, Zimmer Biomet, Arthrex, Conmed, Newclip. Other relationships: All authors have declared that there are no other relationships or activities that could appear to have influenced the submitted work.

\section{References}

1. Hjelle K, Solheim E, Strand T, Muri R, Brittberg M: Articular cartilage defects in 1,000 knee arthroscopies . Arthroscopy. 2002, 18:730-4. 10.1053/jars.2002.32839

2. Widuchowski W, Widuchowski J, Trzaska T: Articular cartilage defects: study of 25,124 knee arthroscopies. Knee. 2007, 14:177-82. 10.1016/j.knee.2007.02.001

3. Ulrich-Vinther M, Maloney MD, Schwarz EM, Rosier R, O'Keefe RJ: Articular cartilage biology. J Am Acad Orthop Surg. 2003, 11:421-30. 10.5435/00124635-200311000-00006

4. Kosy JD, Schranz PJ, Toms AD, Eyres KS, Mandalia VI: The use of radiofrequency energy for arthroscopic chondroplasty in the knee. J Arthrosc Relat Surg. 2011, 27:695-703. 10.1016/j.arthro.2010.11.058

5. Brittberg M, Winalski CS: Evaluation of cartilage injuries and repair. J Bone Joint Surg Am. 2003, 85:58-69. 10.2106/00004623-200300002-00008

6. Sellards RA, Nho SJ, Cole BJ: Chondral injuries. Curr Opin Rheumatol. 2002, 14:134-41. 10.1097/00002281200203000-00010

7. Messner K, Maletius W: The long-term prognosis for severe damage to weight-bearing cartilage in the knee: a 14-year clinical and radiographic follow-up in 28 young athletes. Acta Orthop Scand. 1996, 67:165-8. $10.3109 / 17453679608994664$

8. Lefkoe TP, Trafton PG, Ehrlich MG, Walsh WR, Dennehy DT, Barrach HJ, Akelman E: An experimental model of femoral condylar defect leading to osteoarthrosis. J Orthop Trauma. 1993, 7:458-67. 10.1097/00005131-199310000-00009

9. Montgomery SR, Foster BD, Ngo SS, Terrell RD, Wang JC, Petrigliano FA, McAllister DR: Trends in the surgical treatment of articular cartilage defects of the knee in the United States. Knee Surg Sports Traumatol Arthrosc. 2014, 22:2070-75. 10.1007/s00167-013-2614-9

10. Barber FA, Iwasko NG: Treatment of grade III femoral chondral lesions: mechanical chondroplasty versus monopolar radiofrequency probe. J Arthrosc Relat Surg. 2006, 22:1312-7. 10.1016/j.arthro.2006.06.008

11. Owens BD, Stickles BJ, Balikian P, Busconi BD: Prospective analysis of radiofrequency versus mechanical debridement of isolated patellar chondral lesions. Arthroscopy. 2002, 18:151-5. 10.1053/jars.2002.29906

12. Horton D, Anderson S, Hope NG: A review of current concepts in radiofrequency chondroplasty . ANZ J Surg. 2014, 84:412-6. 10.1111/ans.12130

13. Spahn G, Fröber R, Linss W: Treatment of chondral defects by hydro jet. Results of a preliminary scanning electron microscopic evaluation. Arch Orthop Trauma Surg. 2006, 126:223-7. 10.1007/s00402-005-0002-8

14. Stalder KR, McMillen DF, Woloszko J: Electrosurgical plasmas. J Phys D Appl Phys. 2005, 38:1728. 10.1088/0022-3727/38/11/014

15. Gharaibeh M, Szomor A, Chen DB, MacDessi SJ: A retrospective study assessing safety and efficacy of bipolar radiofrequency ablation for knee chondral lesions. Cartilage. 2018, 9:241-7. 10.1177/1947603517703731

16. Voss JR, Lu Y, Edwards RB, Bogdanske JJ, Markel MD: Effects of thermal energy on chondrocyte viability . Am J Vet Res. 2006, 67:1708-12. 10.2460/ajvr.67.10.1708

17. Meyer ML, Lu Y, Markel MD: Effects of radiofrequency energy on human chondromalacic cartilage: an assessment of insulation material properties. IEEE Trans Biomed Eng. 2005, 52:702-10. 10.1109/TBME.2005.845155

18. Spahn G, Hofmann GO, von Engelhardt LV: Mechanical debridement versus radiofrequency in knee chondroplasty with concomitant medial meniscectomy: 10-year results from a randomized controlled study. Knee Surg Sport Traumatol Arthrosc. 2016, 24:1560-8. 10.1007/s00167-015-3810-6

19. Spahn G, Klinger HM, McKley T, Hofmann GO: Four-year results from a randomized controlled study of knee chondroplasty with concomitant medial meniscectomy: mechanical debridement versus radiofrequency chondroplasty. J Arthrosc Relat Surg. 2010, 26:73-80. 10.1016/j.arthro.2010.02.030

20. Spahn G, Kahl E, Mückley T, Hofmann GO, Klinger HM: Arthroscopic knee chondroplasty using a bipolar radiofrequency-based device compared to mechanical shaver: results of a prospective, randomized, controlled study. Knee Surg Sport Traumatol Arthrosc. 2008, 16:565-73. 10.1007/s00167-008-0506-1

21. Arthroscopic radiofrequency chondroplasty for discrete chondral defects of the knee . (2014). Accessed: December 1, 2020: https://www.nice.org.uk/guidance/ipg493.

22. Lu Y, Edwards RB, Nho S, Cole BJ, Markel MD: Lavage solution temperature influences depth of chondrocyte death and surface contouring during thermal chondroplasty with temperature-controlled monopolar radiofrequency energy. Am J Sports Med. 2002, 30:667-73. 10.1177/03635465020300050601

23. Cetik O, Cift H, Comert B, Cirpar M: Risk of osteonecrosis of the femoral condyle after arthroscopic chondroplasty using radiofrequency: a prospective clinical series. Knee Surg Sport Traumatol Arthrosc. 2009, 17:24-9. 10.1007/s00167-008-0604-0

24. Amiel D, Ball ST, Tasto JP: Chondrocyte viability and metabolic activity after treatment of bovine articular cartilage with bipolar radiofrequency: an in vitro study. J Arthrosc Relat Surg. 2004, 20:503-10. 10.1016/j.arthro.2004.03.018

25. Cameron ML, Briggs KK, Steadman JR: Reproducibility and reliability of the Outerbridge classification for grading chondral lesions of the knee arthroscopically. Am J Sports Med. 2003, 31:83-6. 10.1177/03635465030310012601

26. Pro SL, Blatz BW, McAdams TR, Mandelbaum BR: Chondropenia Severity Score: an arthroscopic stratification tool of structural cartilage changes in the knee as correlated to Patient Reported Outcomes (SS-28). Arthroscopy. 2012, 28:16. 10.1016/j.arthro.2012.04.088

27. Yasura K, Nakagawa Y, Kobayashi M, Kuroki H, Nakamura T: Mechanical and biochemical effect of 


\section{Cureus}

monopolar radiofrequency energy on human articular cartilage: an in vitro study. Am J Sports Med. 2006, 34:1322-7. 10.1177/0363546506287742

28. Uthmanthil RK, Edwards RB, Lu Y, Manley PA, Athanasiou KA, Markel MD: In vivo study on the short-term effect of radiofrequency energy on chondromalacic patellar cartilage and its correlation with calcified cartilage pathology in an equine model. J Orthop Res. 2006, 24:716-24. 10.1002/jor.20108

29. Voloshin I, Morse KR, Allred CD, Bissell SA, Maloney MD, DeHaven KE: Arthroscopic evaluation of radiofrequency chondroplasty of the knee. Am J Sports Med. 2007, 35:1702-7. 10.1177/0363546507304328

30. Carr A: Arthroscopic surgery for degenerative knee. BMJ. 2015, 350:2983. 10.1136/bmj.h2983 